\title{
Tensioned Metastable Fluid Detectors in Nuclear Security for Active Interrogation of Special Nuclear Materials-Part B
}

\author{
Jeffrey A. Webster*, Rusi P. Taleyakhan \\ School of Nuclear Engineering 400 Central Drive, Purdue University, West Lafayette, USA \\ E-mail: jawebste@purdue.edu,rusi@purdue.edu \\ Received June 22, 2011; revised July 25, 2011; accepted August 31, 2011
}

\begin{abstract}
This paper (constituting Part B) addresses active interrogation for detecting Special Nuclear Materials (SNMs) and includes description of the transformational Tensioned Metastable Fluid Detector (TMFD) based method for optimal monitoring. One of the greatest difficulties in detection of SNMs by active interrogation is the task of distinguishing between the probing particles and the secondary particles that indicate the presence of SNMs. The TMFD's selective insensitivity and $\gamma$ photon blindness features are advantageous for alleviating this problem. The working principle of the TMFD is discussed along with its applications for security. The experimental work to date involving detection of small quantities of uranium with conventional detectors is discussed along with results of fission neutron detection. Statistically significant detection was achieved within 5 minutes of counting to ascertain and measure conclusive evidence for the presence of a 55 g sample of uranium containing $<0.1 \mathrm{~g}$ of ${ }^{235} \mathrm{U}$. Results of simulations of three active detection techniques utilizing a TMFD system are presented. The process for using the TMFD to discriminate active source particles using timing and energy are described. These simulations indicate that it should be possible to utilize the TMFD system for optimal neutron-based interrogation of SNMs.
\end{abstract}

Keywords: Tensioned Metastable Fluid Detector, Active Interrogation, TMFD, SNM Detection

\section{Introduction}

This paper describes the process and potential benefits of detection of special nuclear materials (SNMs) by use of active neutron-based interrogation, including use of conventional and novel TMFD technology [1]. Several of the challenges facing detection of fissionable materials will be addressed and the use of this technique for nuclear security applications is described. Active interrogation [2] is advantageous for detection of $U$ (fissionable and fissile) based materials because there are many such materials that emit negligibly low radiation on their own and therefore can be effectively shielded. Passive detector systems can become incapable for nuclear security-related monitoring if the SNM is cleverly hidden by a determined adversary. This comprises a nuclear security issue at large that, fortunately, may be possible to overcome with an optimally derived solution-one which combines use of an interrogating source (such as neutrons or photons) to induce nuclear reactions in deeply buried (or cloaked) SNMs and thereby, deriving a strong enough characteristic signal which can be monitored with a detector that is optimal for that situation. The TMFD system when combined with such interrogation appears to offer unique advantages to promote nuclear security to the next level. The principle of SNM detection via active interrogation is discussed first, followed with description of experiments specifically configured and successfully conducted for detecting minute quantities of fissile uranium, followed thereafter, with description of the TMFD technology accompanied with adaptation for active interrogation.

Active interrogation bombards the SNM with nuclear particles that lead to fission and the release of energetic secondary particles such as fast neutrons (both prompt and delayed) as well as energetic $\gamma$ photons which can then provide information on the quantity and type of the SNM. Detection of these secondary particles is a means for finding illicit SNMs that could be used for the purpose of combating terrorism [2], as well a means to mon- 
itor for the diversion of SNMs like Pu during spent nuclear fuel reprocessing.

A principal challenge involved in detection of SNMs by active interrogation is the task of distinguishing between the probing particles and the secondary particles that indicate the presence of SNMs. This becomes increasingly problematic when attempting to detect highly enriched uranium (HEU) which, by itself emits negligible penetrating radiation such as fast neutrons or $\mathrm{MeV}$ photons. As such, besides swipe tests for $\alpha$ activity, active interrogation is the only way known to be effective for well-shielded HEU. However, scanning thick sections requires an intense quantity of particles from the particle generator, which can often electronically saturate conventional detectors during critical periods. Selective insensitivity [1] in a detector (to $\gamma$ photons) is highly desirable to avoid such dead time issues.

TMFDs are versatile in that they can be configured to spectroscopically detect a wide variety of particles while remaining insensitive to others $[1,3]$. For the application of active interrogation, a TMFD has two advantages. First it can be made selectively "blind" to $\gamma$ photon radiation [4], which can readily overcome and saturate conventional (e.g., NE-213, ${ }^{3} \mathrm{He}, \mathrm{LiI}_{1} \mathrm{BF}_{3}$ ) detectors. Secondly, a TMFD can be cycled on and off in a controlled fashion within microseconds [1] in order to detect secondary particles from interrogation while avoiding the probing particles altogether allowing measurement of small fluxes that normally would be masked by the interrogation particles.

For this application, an Acoustically Tensioned Metastable Fluid Detector (ATMFD), under development at Purdue University, uses focused acoustic waves to create tailored time-varying tension and compression states in a liquid. Nuclear particle interactions with a sufficiently tensioned metastable liquid result in small vapor explosions that are both visible and audible. Neutrons are detected through two interaction types. Fast neutrons are detected through elastic scattering. Thermal neutrons are detected [1] by absorption and then emission of a secondary particle, such as an $\alpha$ particle or heavy ion, which then deposits its energy in the liquid and results in transient bubble nucleation.

Since acoustic waves are used to tension the liquid, the detector alternates between negative and positive pressures which results in it being alternately sensitive and insensitive to neutrons. This fluctuation in sensitivity allows differentiation between the primary and secondary particles in neutron-induced active interrogation. In addition to using timing to discriminate source particles, the energy threshold nature of detection in a TMFD could be used to ignore lower energy particles from a source and only detect higher energy fission neutrons. Both approaches were investigated using simulations and their results are summarized herein.

A $14 \mathrm{MeV}$ pulsed neutron generator (PNG) could be used to probe the sample of interest. The generation of neutrons would be phase locked with the ATMFD so that neutrons are generated while the detector liquid is under positive pressure which makes it blind to all nuclear particles. Since the detector is insensitive to the PNG neutrons during the positive pressure state, only those prompt neutrons and delayed neutrons resulting from fission during times when the detector will "see" them are detected while the TMFD is in tension states of metastability. As the sample is irradiated, delayed neutron precursors accumulate and result in an increasing (eventually reaching an equilibrium level) delayed neutron flux passing through the detector.

This method of detection of fissionable materials is advantageous for security applications for many reasons. First, the ATMFD can be made selectively "alwaysblind" to $\gamma$ photon radiation; therefore, prompt and delayed activation $\gamma$ photons created by neutron irradiation are ignored. This also prevents a fissionable material from being masked by $\gamma$ photon emitters, such as medical waste. Secondly, while operating on a sample with no fissionable material or neutron background, the detector exhibits no response; therefore it results in a fool proof method to determine the presence of various SNMs of interest. Also, if a photo-fission method is used, the ATMFD would once again not suffer from saturation by the photon beam used to interrogate the SNM targets. Lastly, the uniquely simple and straightforward operating mechanism [1] of the TMFD system does not require a large accompanying system of electronics to intemperate detection events.

The purpose of the research work presented herein, is to investigate and demonstrate the prospect of detecting fission neutrons from the active neutron based fission of SNMs, and to remain insensitive to the other particles which may saturate state-of-the-art detection systems, thereby, presenting a major advancement for active SNM detection systems (either neutron and photon-based).

The first step in the process of developing and demonstrating the unique attributes of this new detection system has been to first compare versus conventional detectors to detect the presence of fissionable material. This was done for two reasons. First it had to be determined that small amounts of uranium could indeed be detected with an optimized interrogation-cum-geometry based techniques using conventional detectors, thereby, providing for an advance in this conventional arena as well. Secondly, the eventual usefulness of the ATMFD system is dependent on its ability to outperform these conventional detectors, so the ATMFD's operation and abilities needed to be compared against the baseline as assessed. 
In addition to experimentation with conventional detectors, simulations of the neutron transport from source, to sample, to detector have been conducted to predict the particle fluence required to use an ATMFD for active detection using various techniques. These are presented later in section 3.2.

\section{Experimental Work}

Experimental work was first conducted to develop and to demonstrate the ability to detect small samples of uranium using conventional detectors, as a preparation of the framework for performing such interrogation with TMFDs.

\subsection{Experimental Setup and Procedure}

In order to assess the utility of conventional detectors for neutron based active interrogation, a series of experiments were conducted in which a small sample of uranium was fissioned and detected. The experiments conducted have used a $1 \mathrm{Ci}^{239} \mathrm{Pu}$ based Plutonium-Beryllium (Pu-Be) neutron source (emitting $\sim 2 \times 10^{6} \mathrm{n} / \mathrm{s}$ ) to induce fission in a $55 \mathrm{~g}$ sample of Uranyl Nitrate (UN) containing $\sim 55 \mathrm{~g}$ of ${ }^{238} \mathrm{U}$ and $<0.1 \mathrm{~g}$ of ${ }^{235} \mathrm{U}$. This was followed by a second experiment with $\mathrm{a} \sim 630 \mathrm{~g}$ sample of natural uranium in the form of four $\mathrm{UO}_{2}$ rods. Both samples were to be detected with the same procedure so only the process for experiment design for detecting $55 \mathrm{~g} \mathrm{UN}$ sample is explained.

The goal of this exercise was to design and develop a suitable configuration and protocol for demonstrating rapid detection of such small quantities of $U$ with common laboratory-scale isotope neutron sources like $\mathrm{Pu}-\mathrm{Be}$ and ${ }^{252} \mathrm{Cf}$ using conventional detectors. For neutron detection which was the main focus of the work, a $100 \mathrm{cc}$ Eljen ${ }^{\mathrm{TM}}$ Liquid Scintillation (LS) Detector using NE-213 ${ }^{\mathrm{TM}}$ scintillation liquid was used. A Saint-Gobain/ Bicron, Inc. Sodium-Iodide (NaI) detector was also used to observe and provide supporting confirmation on $\gamma$ photon activity. In order to increase the probability of distinguishing between source and fission neutrons, the experimental configuration was optimized to shield the detectors from the neutron- $\gamma$ source while keeping them in direct line of sight with the uranium sample being interrogated. After some iteration, the geometry shown in Figure 1, and Figure 2 was devised.

The $1 \mathrm{Ci} \mathrm{Pu}-\mathrm{Be}$ neutron source emits roughly equal quantities of neutrons and $\gamma$ photons amounting to an emission intensity of $\sim 2 \times 10^{6} \mathrm{n}-\gamma / \mathrm{s}$. In order to provide shielding for the (NaI and LS) detectors from the source, a loss in intensity to the sample must be incurred because of increased distance. Since the LS detector is practically

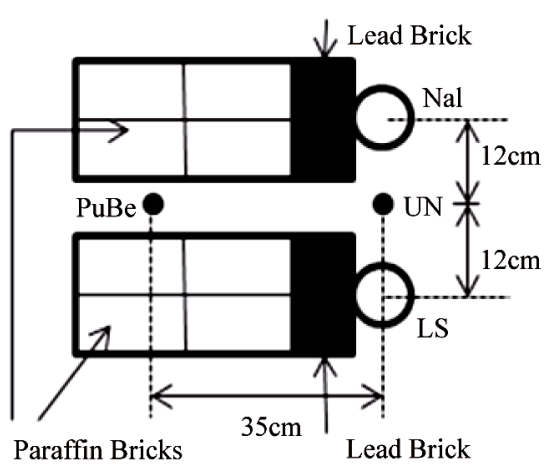

Figure 1. Experimental geometry for fission of a uranium sample. Paraffin and lead bricks are used for neutron and photon shielding, respectively.

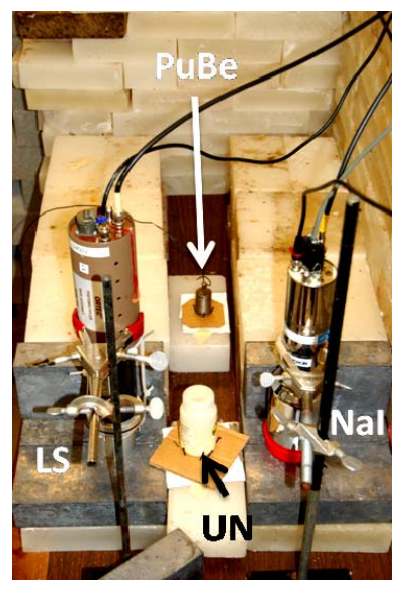

Figure 2. Photograph of experimental setup with Uranyl Nitrate, PuBe source, and Detectors.

capable of monitoring for neutrons only above $\sim 0.5$ $\mathrm{MeV}$, the shielding needs only to down scatter the $(\sim 4$ $\mathrm{MeV}$ average energy) $\mathrm{Pu}-\mathrm{Be}$ source neutrons, not stop them altogether. The paraffin shielding cuts down some of the source neutrons and the lead helps to reduce the $\mathrm{Pu}$-Be source's $4.4 \mathrm{MeV} \gamma$ photons from reaching the detectors, the goal being to minimize the background against which reliable fission-induced detection of $U$ atoms is to be attained with minimal detection time.

Since the LS detector is also sensitive to $\gamma$ photons in addition to neutrons, pulse shape discrimination (PSD) was used, in order to filter out $>99 \%$ of the $\gamma$ photons without a significant loss of neutron counts in the overlap region. Since such a PSD will also filter neutrons, a favorable discrimination level was found through systematic experimentation. Two Spectrum Techniques, Inc. $\mathrm{UCS} 20^{\mathrm{TM}}$ multi-channel analyzers (MCAs) recorded the pulse-height energy spectra from the LS and NaI detectors.

The two detectors were calibrated with two $\gamma$ photon sources, ${ }^{60} \mathrm{Co}$ and ${ }^{137} \mathrm{Cs}$. Once the detectors were cali- 
brated, the PSD was set up for the LS detector. A Time to Amplitude Converter/Single Channel Analyzer (TAC/ SCA) was used to discriminate pulses from the LS detector based on decay pulse timing. Since photons will generally have a shorter pulse decay time than neutrons in the LS detector, a lower level discriminator (LLD) was set on the TAC/SCA to block as many $\gamma$ photons as possible while allowing passage to neutrons. To test the effectiveness of the discrimination setting, the two $\gamma$ photon sources and the UN sample were measured by the LS for 60 seconds with and without PSD. The results of this are shown in Table 1.

It is seen from Table 1 that the $\gamma$ photons coming from the UN sample are cut down by over $99 \%$.

Once the PSD was set up the active neutron-induced interrogation experiment could begin. After acquiring a background count of the environment without the $\mathrm{Pu}-\mathrm{Be}$ or UN sources, the UN bearing sample was next measured by itself so that background from the sample could be subtracted from the final measurement. Next, a measurement was taken with only the $\mathrm{Pu}-\mathrm{Be}$ source in place to subtract the source particles from the irradiation measurement. Next, a measurement with both the source and sample was taken. Lastly, another measurement of just the UN sample was taken to make sure that it hadn't significantly increased in total activity due to neutron irradiation. The measurements of the source and the UN sample were subtracted from the measurement with both source and sample to get a count of the fission-induced neutrons. The two measurements of the sample were averaged for the final calculation. This process was repeated several times in order to better derive confidence of the statistical significance of the measurements.

In addition to repeated testing, the statistical significance of our data was improved further by dividing the energy spectrum into segments and measuring them separately, because it was found that the lower energy channels of the MCA exhibit greater counting efficiency with uranium present. Four energy segments were measured several times each, which required $\sim 4$ hours of total counting time to achieve improved statistics.

Each neutron measurement was also simultaneously matched by a photon measurement from the $\mathrm{NaI}$ detector. The photon measurements were also used to evaluate the effectiveness of a standard $\gamma$ photon detector for measuring fission induced $\gamma$ photons in this setup.

Table 1. LS 60 second $\gamma$ photon counts with and without PSD.

\begin{tabular}{cccc}
\hline Source & PSD OFF & PSD ON & $\% \gamma$ Rejection \\
\hline $\mathrm{UN}$ & 2281 & 10 & 99.6 \\
${ }^{60} \mathrm{Co}$ & 82740 & 260 & 99.7 \\
${ }^{137} \mathrm{Cs}$ & 82100 & 504 & 99.4 \\
\hline
\end{tabular}

\subsection{Neutron Detection Results}

As mentioned earlier, utilizing PSD, $\sim 99 \%$ of the $\gamma$ photons were rejected. It was confirmed that there is a larger effect in the lower energy channels (i.e., as to be expected for a fission neutron spectrum) due to which focus was on 102 of the energy channels out of 256. An example set of measurements for the lowest energy segment in shown in Table 2.

The data presented in Table 2 reports the number of neutron-related signals measured in energy (pulse height spectrum) channels 5-24. Each measurement was 300 seconds long. Once these measurements were completed, they were repeated for pulse height spectra channels 25-51, 52-76, and 77-102. The results for the four energy ranges are shown in Table 3 .

Table 2. Neutrons counted in 300 seconds in MCA channels 5-24.

\begin{tabular}{|c|c|c|c|c|c|}
\hline Trial & UN & $\mathrm{Pu}-\mathrm{Be}$ & $\mathrm{UN}+\mathrm{Pu}-\mathrm{Be}$ & Difference $^{+}$ & $\hat{\sigma}$ \\
\hline Before & 37 & $\mathrm{x}$ & $\mathrm{x}$ & $\mathrm{x}$ & $\mathrm{x}$ \\
\hline 1 & $\mathrm{x}$ & 6466 & 7041 & 535 & 4.60 \\
\hline 2 & $\mathrm{x}$ & 6743 & 7036 & 253 & 2.16 \\
\hline 3 & $\mathrm{x}$ & 6541 & 7091 & 510 & 4.37 \\
\hline After & 43 & $\mathrm{x}$ & $\mathrm{x}$ & $\mathrm{x}$ & $\mathrm{x}$ \\
\hline Total & $120^{*}$ & 19750 & 21168 & 1298 & 6.42 \\
\hline
\end{tabular}

Table 3. Neutrons counted in 900 seconds in several sets of MCA channels.

\begin{tabular}{ccccccc}
\hline $\begin{array}{c}\text { Lower } \\
\text { Limit }\end{array}$ & $\begin{array}{c}\text { Upper } \\
\text { Limit }\end{array}$ & UN & Pu-Be & UN + Pu-Be Difference & $\hat{\sigma}^{\wedge}$ \\
\hline Channel & Channel & $\begin{array}{c}\text { Total } \\
\text { Counts }\end{array}$ & $\begin{array}{c}\text { Total } \\
\text { Counts }\end{array}$ & Total Counts & & \\
\hline 5 & 24 & 120 & 19750 & 21168 & 1298 & 6.4 \\
25 & 51 & 87 & 30195 & 31800 & 1518 & 6.1 \\
52 & 76 & 62 & 19952 & 21026 & 1013 & 5.0 \\
77 & 102 & 21 & 13236 & 13714 & 457 & 2.8 \\
\hline
\end{tabular}

${ }^{+}$This difference is $(\mathrm{UN}+\mathrm{Pu}-\mathrm{Be})-(\mathrm{Pu}-\mathrm{Be})-(\mathrm{UN}) ;{ }_{1} 1 \sigma=\sqrt{\mathrm{Pu}-\mathrm{Be}+(\mathrm{UN}+\mathrm{Pu}-\mathrm{Be})}$. 
While statistically significant results are obtained for all four energy ranges, there are diminishing returns as one goes to higher channels, which is to be expected because of the declining detector efficiency with increased energy and also the fact that a lesser quantity of higher energy neutrons emanate from fission compared to lower energies.

The same procedure described in section 2.1 was utilized for interrogation of a larger uranium sample $(\sim 630$ $\mathrm{g}$ of natural $\mathrm{UO}_{2}$ in the form of four fuel rods). With the same experimental setup and the relatively larger amount of uranium, much fewer measurements were required. The results of monitoring are shown in Table 4.

The $\mathrm{UO}_{2}$ sample was more readily detectable in the same configuration as the UN. It should be noted that the only difference in the procedure was the channel window used for these measurements (5-102) was measured all at once instead of being broken up into four measurements as in Table 3. This was done because, when interrogating $630 \mathrm{~g}$ of $\mathrm{UO}_{2}$ vs. $55 \mathrm{~g}$ of $\mathrm{UN}$, breaking up the measurements into separate energy regions was not necessary to get good-enough statistics and the data could be acquired faster.

While it was initially obvious that the presence of the $\mathrm{UO}_{2}$ sample had an effect on the count rate, to verify that indeed fission neutrons are being measured, the energy spectrum of the measured neutrons was compared to that of the known fission spectrum of a ${ }^{252} \mathrm{Cf}$ spontaneous fission neutron source. This was done by calculating differences in each energy bin with and without the $\mathrm{UO}_{2}$ sample and plotting the resulting spectrum against a ${ }^{252} \mathrm{Cf}$ based measurement, and also from a $\mathrm{Pu}-\mathrm{Be}$ source measurement made from the same detector with the same settings on the same day. The neutron energy spectrum was compared to that of the ${ }^{252} \mathrm{Cf}$ and PuBe spectra by organizing the counts into a histogram with bins made up of five MCA channels each. The spectra were normalized by dividing each bin by the sum of the entire spectrum. The results are shown in Figure 3.

It is obvious that the $\mathrm{Pu}-\mathrm{Be}$ source neutron-interrogated $\mathrm{UO}_{2}$ sample produced in turn neutrons which

Table 4. Neutrons counted in LS detector from $\mathrm{UO}_{2}$ sample in 300 seconds in MCA channels 5-102.

\begin{tabular}{cccccc}
\hline Test & $\mathrm{UO}_{2}$ & $\mathrm{PuBe}$ & $\mathrm{UO}_{2}$ and PuBe & Difference & $\sigma$ \\
\hline 1 & 563 & 11872 & 13636 & 1201 & 7.52 \\
2 & 597 & 11819 & 13375 & 959 & 6.04 \\
3 & 649 & 11743 & 13360 & 968 & 6.11 \\
4 & 719 & 11749 & 13393 & 925 & 5.83 \\
5 & 817 & 12473 & 13631 & 341 & 2.11 \\
Total & 3345 & 59656 & 67395 & 4394 & 12.3 \\
\hline
\end{tabular}

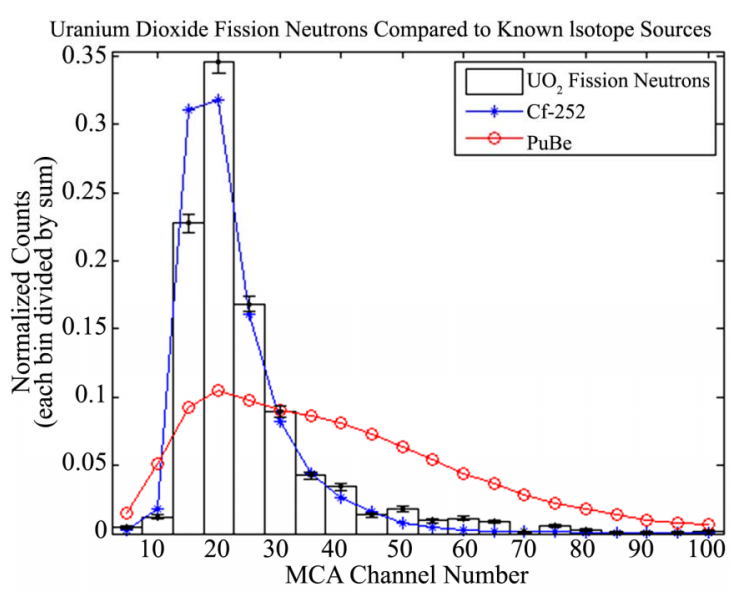

Figure 3. $\mathrm{UO}_{2}$ fission spectrum plotted as a histogram with 5 MCA channels per bin. Also plotted are the ${ }^{252} \mathrm{Cf}$ and $\mathrm{Pu}-\mathrm{Be}$ spectrums for comparison. Error bars are $1 \sigma$ and are too small to see for the ${ }^{252} \mathrm{Cf}$ and $\mathrm{Pu}-\mathrm{Be}$ points.

match the neutron energy spectrum of the ${ }^{252} \mathrm{Cf}$ fission source very well. The larger uranium sample made it far easier to identify the fission spectrum coming from it. It should be noted that both the data from the $\mathrm{UN}$ and $\mathrm{UO}_{2}$ experiments have about the same number of detection events $(\sim 4300)$, but since the $\mathrm{UO}_{2}$ data was taken over a much shorter time span, the effect of the photon and neutron background was minimized. The slight discrepancy in the lower energy channels is attributed to electronic limitations of the pulse shape discrimination process. When the pulse widths of detection events in the LS detector are plotted, there is some overlap between the high energy photons and low energy neutrons. As a result one cannot fully reject all photons due to which loss of some lower energy neutrons will occur. With that said, the curves in Figure 3 make it obvious that, using the as-designed approach along with the developed protocol for active neutron based interrogation, neutrons resulting from fission were convincingly detected from a $600 \mathrm{~g}$ $\mathrm{UO}_{2}$ target within only $5 \mathrm{~min}$, using a common liquid scintillation detector (upon interrogating the uranium sample with a $1 \mathrm{Ci} \mathrm{Pu}-\mathrm{Be}$ neutron source of a different energy spectrum).

\subsection{Photon Detection Results}

It was quickly noticed that the $\gamma$ photon counts from the UN sample alone increased significantly upon irradiation from the $\mathrm{Pu}-\mathrm{Be}$ source. As a result of this, the $\mathrm{NaI} \gamma$ photon measurements taken directly with the neutron measurements were not as conclusive. To resolve this issue, separate experiments were conducted to measure $\gamma$ photons from the Uranium fission. The experimental setup and calibration was the same as in section 2.1 , with 
one exception being that the LS detector was gated for only $\gamma$ photon counting. Filtering out the neutrons on the LS was done by setting the discriminator level on the TAC/SCA in the opposite configuration from that for neutron detection. The level at which the lower level discriminator had been set for neutron counting was now the setting for the upper level discriminator, which resulted in the opposite effect.

With the LS and NaI detectors both simultaneously measuring photons, a series of tests were conducted to monitor $\gamma$ photons resulting from neutron interrogation of UN. After observing that a long set of repeated measurements resulted in the $\gamma$ photon background in the UN sample increasing dramatically, it was decided that single shorter measurements would be more beneficial. Shown in Table $\mathbf{5}$ are some examples of the single trials.

It can be seen in Table 5 that doing a single measurement for 300 seconds with the $\mathrm{NaI}$ detector can result in a significant increase in $\gamma$ photon counts attributed to fission. A measurement of 60 seconds with the LS detector resulted in a similar but lesser significant result as may be expected since the detection efficiency for photons is much lower than for a $\mathrm{NaI}$ detector. Also, notice that the counts per minute in the difference column of the LS detector is considerably higher $(\sim 10 \gamma / \mathrm{s})$ than the neutron count rate of $\sim 4 \mathrm{n} / \mathrm{s}$ for the LS detector from the data of Table 4, which is as it should be, since the emanations are due to U-fission. In summary, it is demonstrated that a change in photons resulting from fission can also be measured with the LS detector, but the neutron measurements are more conclusive.

Table 5. NaI and LS photon measurement.

\begin{tabular}{ccccccc}
\hline Det. & Time $(\mathrm{sec})$ & $\mathrm{UN}$ & $\mathrm{Pu}-\mathrm{Be}$ & $\mathrm{UN}+\mathrm{Pu}-\mathrm{Be}$ & Diff & $\sigma$ \\
\hline $\mathrm{NaI}$ & 300 & 116304 & 1027245 & 1170827 & 27278 & 18.4 \\
$\mathrm{LS}$ & 60 & 2481 & 6412 & 9460 & 567 & 4.5 \\
\hline
\end{tabular}

\section{ATMFD Active Interrogation System}

In this section we discuss the various attributes associated with utilizing the ATMFD in conjunction with active neutron based interrogation of SNMs.

\subsection{ATMFD Hardware}

The hardware typically used to operate an ATMFD is shown in Figure 4.

As shown in Figure 4, the signal from the waveform generator is sent through the amplifier and provides power for the acoustic driver. The oscilloscope records

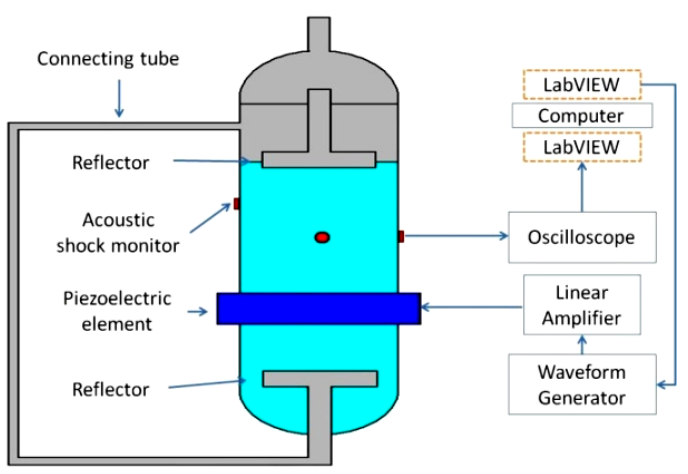

Figure 4. Hardware wiring diagram for ATMFD [5].

the shock pulses from detection events and data analysis is done with a LabVIEW ${ }^{\mathrm{TM}}$ program. If provided with a power measurement, the LabVIEW ${ }^{\mathrm{TM}}$ program [5] can also automatically find the resonance frequency. This is important because the PNG neutron source will need to be automatically controlled as well.

\subsection{Active Interrogation Using ATMFD}

Several techniques are described herein to utilize the advantages of the ATMFD system for active interrogation. The goal is to use the ATMFD's capabilities to become blind to the source particles from any active interrogating source. Two main approaches for discriminating source particles have been simulated. The first approach is using a pulsed $14 \mathrm{MeV}$ neutron source timed to the acoustic cycle of the ATMFD. The other option investigated was of lower energy of $2.45 \mathrm{MeV}$ and $60 \mathrm{keV}$, respectively.

In order to generate neutrons that go undetected in the ATMFD, one way is to have the neutrons pass through the detector when it is under positive pressure. This can be done with a pulsed neutron generator (PNG) system such as a $14 \mathrm{MeV}$ D-T fusion neutron generator. The PNG is controlled by $5 \mathrm{~V}$ gate pulses which offer the user timing control as to when neutrons are generated. Properly timed trigger pulses can be used to make the PNG phase-lock with the waveform generator so that the signals are of the type shown in Figure 5. Such phase-locking has

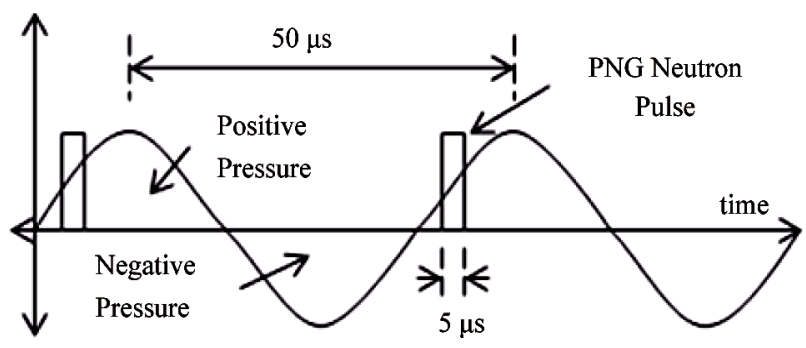

Figure 5. Acoustic driver (sine wave) and PNG gate (square wave) signals. 
been successfully demonstrated [6].

Figure 5 shows a specific sample of control signals for the ATMFD acoustic driver and the PNG. The PNG pulse is phase locked with the sine wave and the phase offset is such that neutrons pass through the detector during the ATMFD's positive pressure cycle. This method prevents detection of the PNG pulsed neutrons, and only the delayed neutrons resulting from fission will be detected by the ATMFD.

Another approach to attain source particle discrimination is to use lower energy neutrons for the source and detecting fission-induced neutrons above that energy. One option for a neutron source is $2.45 \mathrm{MeV}$ neutrons from a D-D fusion neutron generator. The source neutrons have sufficient energy to induce fission in SNMs like U-235 and also in the non-fissile U-238 isotope; it is estimated that $\sim 29 \%$ of the fission energy spectrum is comprised of neutrons of above $2.45 \mathrm{MeV}$ energy. For this approach to work, the detector used must have very effective energy discrimination capabilities, which, indeed, the ATMFD does very well. Since detection in a metastable fluid detector is a threshold reaction based on the liquid tension, energy discrimination of neutrons in a low level discrimination mode is straightforward; i.e., by simply adjusting the tension such that source neutrons from a $2.45 \mathrm{MeV}$ source are rejected, but induced-fission neutrons (above this level) are not.

An alternative interrogating neutron source uses epithermal neutrons. Accelerator systems have been built [7] which utilize a $2 \mathrm{MeV}$ proton beam on a lithium target to generate $60 \mathrm{keV}$ neutrons. Epithermal neutrons can be used to induce thermal fission only in fissile isotopes. Discrimination of epithermal source neutrons with the ATMFD is straightforward. This technique allows for detection of nearly the entire fission neutron energy spectrum, however only fissile isotopes can be detected. Therefore, the utility of this technique is application dependent and, would require a proton-beam accelerator which can be bulky and increase the cost-cum-complexity.

\subsection{Pulsed Neutron Source Technique Modeling}

Monte-Carlo techniques-based estimations were performed to assess for and optimize the operational characteristics. This was conducted with the well-established Monte-Carlo nuclear particle transport codes MCNP5 [10] and MCNP-PoliMi [8]. Since the uranium samples being scanned in baseline experiments were very small, experimental setups with best case conditions were used. Three active interrogation scenarios were simulated. The first two were the detection of the two uranium samples available to us (i.e., $55 \mathrm{~g}$ of $\mathrm{UN}$ and $630 \mathrm{~g} \mathrm{UO}_{2}$ ). The third was a hypothetical scan of a pipe in a typical spent nuclear fuel commercial reprocessing plant. Each of these simulations was conducted in three steps. First the interrogation source and SNM were simulated in MCNP5 and the fission rate in the SNM was tallied. With the fission rate known, a MATLAB ${ }^{\mathrm{TM}}$ script used the delayed neutron precursor yields, fission rate, and precursor decay rate to determine the delayed neutron output over time. This calculation yielded the equilibrium rate of delayed neutron production, which was used to define the source of a MCNP-PoliMi simulation. The last step was a MCNP-PoliMi simulation of a delayed neutron source and an ATMFD in which the rate of delayed neutron detection in the ATMFD was predicted. This entire process will now be explained in more detail.

The active interrogation simulation of the UN sample will be described first. The first model simulated the $14 \mathrm{MeV}$ D-T source as a point source, and a $55 \mathrm{~g}$ sample of UN located $5 \mathrm{~cm}$ away. The actual UN sample is made up of depleted uranium; this model assumed pure ${ }^{238} \mathrm{U}$. The maximum neutron generation rate of our D-T based PNG is $\sim 10^{8}$ neutrons per second, and so was the intensity of the modeled source. MCNP Ver.5 was used in this study. The MCNP5 model generated and tracked $10^{8}$ neutrons and recorded the number of fissions $(20,342)$ that occurred within the UN sample. From this it is estimated that in one second of irradiation, 20,342 fissions would occur within the Uranium. The prompt neutrons would go unnoticed by the detector since they would pass through at the same time as the source neutrons, so a calculation of the delayed neutron generation rate was needed. Since delayed neutrons are the result of the radioactive decay of certain fission products, a production vs. decay calculation was performed to estimate the delayed neutron production rate over time.

As is common practice, the six delayed neutron group approximation was used for calculating the delayed neutron production. Calculations were performed for each of the six groups independently and then the results were combined. The number of atoms of delayed neutron precursors formed by fission was found by multiplying the fission rate by the yield per fission for each of the six groups. The number of delayed neutrons produced was calculated by multiplying the number of delayed neutron precursors by the decay constant for that group and then by time. This was performed iteratively in three steps.

First, at the beginning of a time step, it was assumed that all of the fissions for that step happened instantaneously. A small time step, one millisecond for example, was used so that this assumption was valid. The fission rate times the precursor yield times the length of the time step gave the number of precursor atoms created in that cycle, which was added to the amount from the previous 
iteration. The neutron generation for that step was then calculated by multiplying the number of precursor atoms by the decay constant and the time step. The number of neutrons generated was saved to an array to be plotted. Finally, the number of decays for that step was subtracted from the number of precursor atoms to find how many were left over. This process was repeated for several hundred seconds of irradiation time to plot the neutron production rate over time.

The plot of the delayed neutron production rates over time for active interrogation of $\mathrm{UN}$ is shown in Figure 6.

It can be seen in Figure 6 that at some point in time equilibrium is reached if the production rate remains the same. Having the delayed neutron output at, or nearly at, equilibrium offers the optimal conditions for detection. Once the equilibrium activities of the six delayed neutron groups were known, a MCNP-PoliMi simulation was conducted in which delayed neutrons were generated within the UN sample and detected in an ATMFD. The geometry for this simulation is shown in Figure 7.

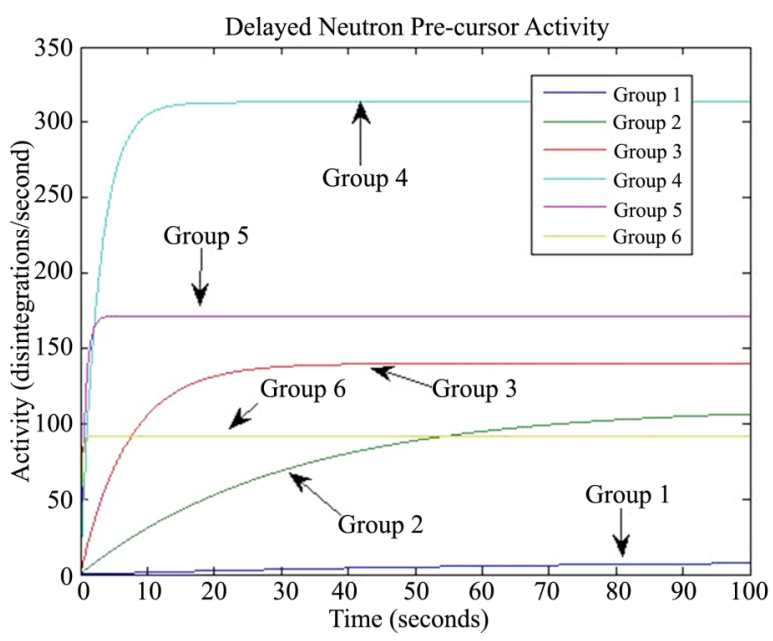

Figure 6. Delayed neutron pre-cursor activity build-up.

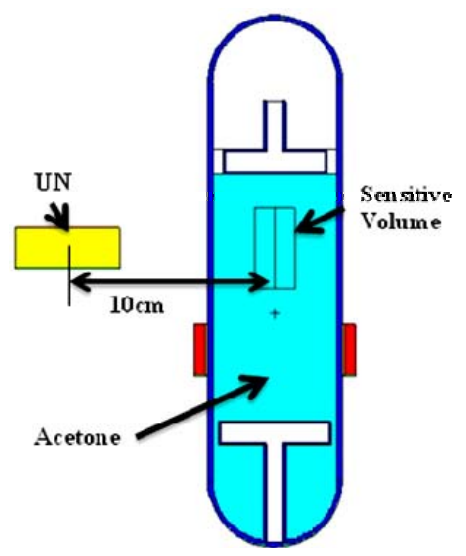

Figure 1. MCNP geometry for the ATMFD to predict delayed neutrons in the sensitive volume.
The sample is located close to the detector to increase the chance of detection. The region labeled sensitive volume represents the volume of liquid in the ATMFD that has been found to detect neutrons reliably in experiments.

The energy of each neutron was the energy of one of the six delayed neutron energy groups. The probability of selecting a neutron from any one group was determined by their relative equilibrium intensities in Figure 6, with group 4 being to most probable. The starting location of each neutron was based on a fission talley from the first MCNP5 simulation in order to better simulate the actual location where it would be generated. This was done by placing a mesh talley over the UN sample in the irradiation simulation to track the fission rate as a function of height in the sample. This resulted in a distribution of fission rates in horizontal slices in the sample. The fission was assumed to be radially and axially symmetric. This vertical distribution was used to assign the location probabilities for generation of delayed neutrons in the latter simulation.

Once the delayed neutron source was properly defined, the next task was defining the detector. Normally, a detector is modeled in MCNP by recording an energy dependent flux talley in the detector volume and then using an external code such as SCINFUL to convert the flux talley to a detection rate, based on the known detection efficiency, at each energy. This can also be done manually. Since no such conversion code or tables exist for the ATMFD, an alternative approach was taken.

Instead of using MCNP5 to simulate delayed neutron detection, MCNP-PoliMi [8] was utilized. For the purpose of this model, MCNP-PoliMi was a better choice because it easily produces far more information about neutron interactions than with MCNP5. The criteria for detection of a neutron in the ATMFD assumed for this study was an elastic scattering event in which a neutron deposits more than $100 \mathrm{keV}$ onto the nucleus of a carbon or oxygen atom within the sensitive volume. MCNPPoliMi will output the location, target nucleus, and energy deposited for every scattering event during the simulation. The output from MCNP-PoliMi was easily imported into a MATLAB ${ }^{\mathrm{TM}}$ based program, where the number of collisions that met the detection criteria was counted.

The neutron generation rate from Figure 6 is 825 neutrons per second and the $100 \mathrm{keV}$ collision rate on carbon and oxygen from MCNP-PoliMi was 0.0187 collisions per second. This means that, under the assumptions made, a detectable collision only occurs every $\sim 54$ seconds. In addition to this, the ATMFD can have at most $50 \%$ intrinsic efficiency, since it spends half of its time insensitive, and will not be fully sensitive when the ten- 
sion is ramping up and down during the negative pressure cycle. By this estimate it would appear that under the conditions that the ATMFD is normally operated, for detection of higher energy and more intense sources, a delayed neutron would only be seen every few minutes.

While this result may be discouraging, there are a number of ways to overcome for faster monitoring. If the tension is increased such that protons from neutron collisions with hydrogen nuclei can also be made to nucleate bubbles, the detection rate will increase by a factor of about 100 . Also more power to the detector can rapidly increase the size of the sensitive volume and therefore allow increased detection. Lastly, selection of an ATMFD fluid such as trimethyl borate which has a high (n,alpha) cross section for low energy neutrons will allow for vastly enhanced detection of the neutrons that were too low in energy to be detected through elastic scattering thereby, bringing down detection time to within seconds.

In addition to simulating active interrogation of the small 55 gram UN sample, two other studies were done, the first of which was a similar model in which $\sim 630$ grams of natural Uranium Dioxide $\left(\mathrm{UO}_{2}\right)$ was interrogated. The real sample, in the lab, is four $\mathrm{UO}_{2}$ rods tightly packed in a canister, and the combined mass of the rods is $\sim 630$ grams. The material was modeled as one single rod with the same height and a radius such that the mass was matched. The same process as the last model was used, in which a $14 \mathrm{MeV}$ point source was $5 \mathrm{~cm}$ away from the $\mathrm{UO}_{2}$. The same source intensity of $10^{8}$ neutrons per second was used. The fission rate from this model was 288,995 fissions per second. After the delayed neutron generation rate was found in MATLAB, the MCNP-PoliMi model shown in Figure 8 was run. The delayed neutron generation rate was 11,689 neutrons per second and the detection rate for delayed neutrons was 0.24 counts per second or once every 4.16 seconds. The full results are also in Table 7 .

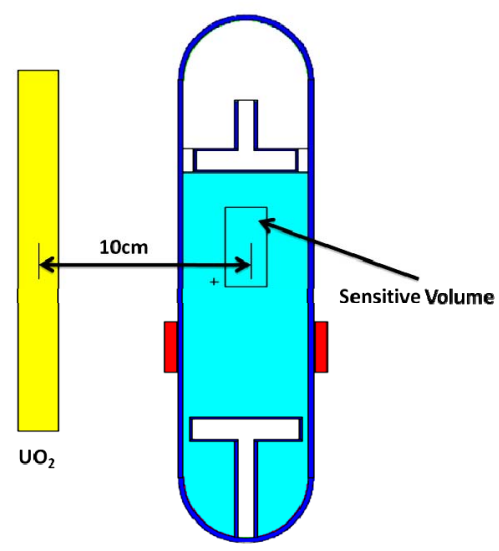

Figure 8. $\mathrm{UO}_{2}$ active interrogation MCNP geometry.
The third scenario modeled was active interrogation of a pipe from a UREX reprocessing stream. This area of modeling is another project within itself, so only one reasonably representative case was chosen to demonstrate the potential. The target is a $1 / 2$ meter long section of $5 \mathrm{~cm}$ inner diameter $1 \mathrm{~cm}$ thick steel pipe filled with UREX feed stream liquid. The composition of the spent fuel was calculated with the well-known ORIGEN code [9] for a PWR core. The UREX liquid modeled was made up of the uranium and plutonium from a $3 \%$, original enrichment, 33GW-day/MTU burn up fuel, after three years of cooling, dissolved in 3.5 Molar nitric acid. Potential neutron contributions from spontaneous fission and $(\alpha, n)$ interactions were modeled separately. The uranium concentration is $200 \mathrm{~g} / \mathrm{L}$ of solution. The relative mass of the fuel used in the model is given in Table 6 [9].

The isotopes used in the model are shown in Table 6. The other fissionable actinides were not modeled because their quantities were several orders of magnitude lower than uranium and plutonium.

Once again the source was $14 \mathrm{MeV}$ neutrons, but this time the source was $10 \mathrm{~cm}$ from the center of the pipe

Table 6. Relative mass of $U$ and Pu from $3 \%$ enriched 33 GW-day/MTU burn up fuel.

\begin{tabular}{cc}
\hline Isotope & Relative Mass \\
\hline & $\mathrm{g} / \mathrm{MTU}$ \\
${ }^{234} \mathrm{U}$ & $1.57 \times 10^{2}$ \\
${ }^{235} \mathrm{U}$ & $7.28 \times 10^{3}$ \\
${ }^{236} \mathrm{U}$ & $3.89 \times 10^{3}$ \\
${ }^{238} \mathrm{U}$ & $9.44 \times 10^{5}$ \\
${ }^{238} \mathrm{Pu}$ & $1.55 \times 10^{2}$ \\
${ }^{239} \mathrm{Pu}$ & $5.45 \times 10^{3}$ \\
${ }^{240} \mathrm{Pu}$ & $2.31 \times 10^{3}$ \\
${ }^{241} \mathrm{Pu}$ & $1.18 \times 10^{3}$ \\
${ }^{242} \mathrm{Pu}$ & $5.47 \times 10^{2}$ \\
\hline
\end{tabular}

Table 7. Results of MCNP $14 \mathrm{MeV}$ pulsed active interrogation simulations

\begin{tabular}{cccccc}
\hline $\begin{array}{c}\text { SNM } \\
\text { Target }\end{array}$ & $\begin{array}{c}\text { Fission } \\
\text { Rate }\end{array}$ & $\begin{array}{c}\text { Delayed } \\
\text { Neutron } \\
\text { Output }\end{array}$ & $\begin{array}{c}>100 \mathrm{keV} \\
\text { Collisions on } \\
\mathrm{C}\end{array}$ & $\begin{array}{c}>100 \mathrm{keV} \\
\mathrm{O}\end{array}$ & $\begin{array}{c}\text { Time } \\
\text { between } \\
\text { Collisions } \\
(1 / \mathrm{C}+\mathrm{O})\end{array}$ \\
\hline & fis/sec & $\mathrm{n} / \mathrm{sec}$ & $\mathrm{col} / \mathrm{sec}$ & $\mathrm{col} / \mathrm{sec}$ & Seconds \\
\hline $\mathrm{UN}$ & 20,342 & $8.25 \times 10^{2}$ & 0.0186 & $4.94 \times 10^{-5}$ & 53.6 \\
$\mathrm{UO}_{2}$ & 288,995 & $1.17 \times 10^{4}$ & 0.239 & $9.35 \times 10^{-4}$ & 4.16 \\
$\mathrm{UREX}^{2}$ & 43,573 & $1.76 \times 10^{3}$ & 0.0141 & $2.30 \times 10^{-2}$ & 61.1 \\
$\begin{array}{c}\text { UREX } \\
\text { Background }\end{array}$ & $6.73 \times 10^{4}$ & 12.6 & 2.9 & 0.0645 \\
\hline
\end{tabular}


because of its increased size. With source strength of $10^{8}$ neutrons per second the fission rate from the section of pipe was 43,573 fissions per second. This resulted in a delayed neutron production rate of 1,761 neutrons per second. The rate of detectable neutron elastic collisions was 0.0164 per second or one every 61 seconds, which is similar to the first case with the UN. The geometry for this simulation is shown in Figure 9.

The results of all three of these simulations are given in Table 7.

Table 7 shows the pulsed active interrogation simulation results. The background was modeled separately and used energy and intensity information based on the output of the well-known ORIGEN code simulation. It is immediately evident that the detection rate is below the predicted background. In order to get a significant count rate scanning the UREX stream, the source intensity would need to be significantly higher than $10^{8} \mathrm{n} / \mathrm{s}$. Since almost all of the background neutrons would be from spontaneous fission of ${ }^{244} \mathrm{Cm}$, this technique would be more useful in a part of the UREX process without $\mathrm{Cu}$ rium present. In application, a much stronger source would be needed to see delayed neutrons, but this model was meant to simulate something that could be recreated in the lab as part of future work, which would have a negligible passive neutron output since the only fissionable material would be uranium.

\subsection{Lower Energy Neutron Source Technique}

As mentioned previously, a couple other techniques

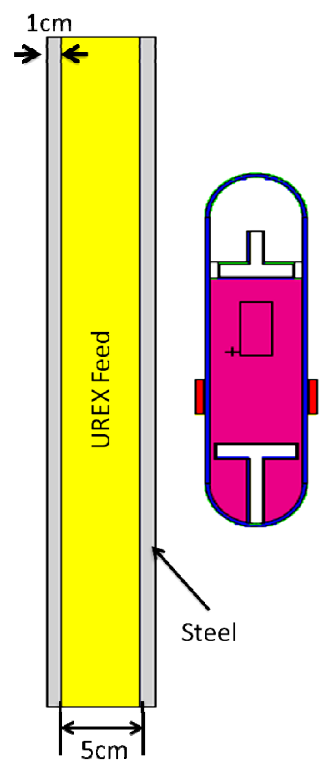

Figure 9. MCNP geometry for active interrogation of UREX feed pipe. for active detection have been simulated which use lower energy neutron sources. MCNP-PoliMi was used to conduct simulations for interrogation of UREX feed liquid with $2.45 \mathrm{MeV}$ and $60 \mathrm{keV}$ sources.

Since the lower energy source techniques use prompt fission neutrons for detection, the entire simulation can be done in one model and delayed neutrons ignored. To simulate a D-D fusion source, the MCNP-PoliMi model with UREX feed solution and the ATMFD was altered to include a $2.45 \mathrm{MeV}$ or $60 \mathrm{keV}$ continuous source instead of $14 \mathrm{MeV}$. For the case of the $2.45 \mathrm{MeV}$ source, an isotropic continuous source of $10^{8} \mathrm{n} / \mathrm{s}$ was modeled $10 \mathrm{~cm}$ from the center of the UREX feed pipe. The $60 \mathrm{keV}$ source was modeled as a mono-directional continuous source with intensity of $5 \times 10^{6} \mathrm{n} / \mathrm{s}$, which agrees with specifications found in literature [7].

To simulate source particle discrimination, the talley recorded for the sensitive volume of the ATMFD was set to only be sensitive to neutrons above $2.5 \mathrm{MeV}$ or 100 $\mathrm{keV}$ depending on the source. By doing this, only neutron collisions in the sensitive volume of the detector with sufficient energy were recorded. The results of the two simulations along with predicted background are given in Table 8.

It can be seen in Table 8 that both the $2.45 \mathrm{MeV}$ and 60 $\mathrm{keV}$ source simulations yielded a signal to background ratio of $\sim 4$. The background for the $2.45 \mathrm{MeV}$ source case would be lower since a large portion of the background would be discriminated along with the source particles. The techniques which utilize detection of prompt neutrons appear to be more efficient; however the preferred technique would be dependent on the application. The epithermal source technique would only be sensitive to fissile material which may or may not be advantageous.

Table 8. Results of lower energy neutron source simulations.

\begin{tabular}{|c|c|c|c|c|c|}
\hline $\begin{array}{l}\text { Neutron } \\
\text { Source }\end{array}$ & $\begin{array}{c}\text { Fission } \\
\text { Rate }\end{array}$ & $\begin{array}{c}\text { Neutron } \\
\text { Output }\end{array}$ & $\begin{array}{c}\text { Detectable } \\
\text { Collisions on } \\
\text { C Atoms }\end{array}$ & $\begin{array}{c}\text { Detectable } \\
\text { Collisions on } \\
\text { O Atoms }\end{array}$ & $\begin{array}{c}\text { Time } \\
\text { between } \\
\text { Collision } \\
(1 / \mathrm{C}+\mathrm{O})\end{array}$ \\
\hline Units & fis/s & $\mathrm{n} / \mathrm{s}$ & $\mathrm{col} / \mathrm{s}$ & $\mathrm{col} / \mathrm{s}$ & seconds \\
\hline $2.45 \mathrm{MeV}$ & 55,064 & 153,971 & 9 & 1 & 0.1 \\
\hline $60 \mathrm{keV}$ & 92,553 & 261,107 & 41 & 15 & 0.0179 \\
\hline $\begin{array}{c}\text { UREX } \\
\text { Background }\end{array}$ & & 67,308 & 12.6 & 2.9 & 0.0645 \\
\hline $\begin{array}{l}\text { Background } \\
>2.45 \mathrm{MeV}\end{array}$ & & 19,338 & 1.9 & 0.3 & 0.4545 \\
\hline
\end{tabular}




\section{Conclusions}

The background theory and technical explanations of the ATMFD have been described. Carefully designed experimental work with conventional detectors has allowed us the ability to detect uranium in significantly smaller quantities than that reported in the literature by others. Detection of both neutrons and photons from the fission of uranium was demonstrated. Simulations of three proposed active detection techniques using the ATMFD have been conducted. Active detection using a pulsed 14 $\mathrm{MeV}$ source was investigated first. Using a $2.45 \mathrm{MeV}$ source and setting the tension level on the ATMFD to discriminate source particles is found to be the most efficient detection method that is not limited to detecting fissile isotopes alone. For interrogating samples with a higher concentration of fissile material, epithermal neutrons would be preferable but would require a separate proton accelerator. These simulations present the potential for the ATMFD system for optimal and superior neutron-based interrogation of SNMs.

\section{Acknowledgements}

This research was sponsored in part by the United States National Science Foundation, Department of Homeland Security, Department of Energy, and Purdue University, State of Indiana. The timely assistance provided by Pur- due University's Radiological and Environmental Ser- vices is acknowledged; also the valuable discussions with B. Archambault, J. Lapinskas, A. Sansone, and M. Ziolkowski of Purdue University, S. McDeavitt and W. Charlton of Texas A \& M Univ., and C. Pereira of Argonne National Lab.

\section{References}

[1] R. P. Taleyarkhan, J. Lapinskas and Y. Xu, "Tensioned Metastable Fluids and Nanoscale Interactions with Exter- nal Stimuli-Theoretical-Cum-Experimental Assessments and Nuclear Engineering Applications," Nuclear Engineering and Design, Vol. 238, No. 7, 2008, pp. 18201827. doi:10.1016/j.nucengdes.2007.10.019

[2] W. L. Myers, et al., "Photon and Neutron Active Interrogation of Highly Enriched Uranium," Americal Institute of Physics Conference Proceedings, Vol. 769, 2005, pp. 1688-1692.

[3] B. Archambault, et al., "Transformational Nuclear Sensors-Real-Time Monitoring of WMDs, Risk Assessment \& Response," IEEE, 2010, pp. 421-427.

[4] A. Sansone, et al., "Gamma-Blind Nuclear Particle-Induced Bubble Formation in Tensioned Metastable Fluids," Proceedings of 2011 Annual American Nuclear Society Annual Conference, Hollywood, USA, June 2011, p. 1033.

[5] J. Lapinskas, et al., "Tension Metastable Fluid Detection Systems for Special Nuclear Material Detection and Monitoring," Nuclear Engineering and Design, Vol. 240, No. 10, 2010, pp. 2866-2871. doi:10.1016/i.nucengdes.2010.05.058

[6] R. P. Taleyarkhan, et al., "Evidence of Nuclear Emissions during Acoustic Cavitation," Science, Vol. 295, No. 5561, 2002, pp. 1868-1873. doi:10.1126/science.1067589

[7] C. A. Hagmann, et al., "Active Detection of Shielded SNM with 60-keV Neutrons," IEEE Transactions on $\mathrm{Nu}$ clear Science, Vol. 56, No. 3, 2009, pp. 1215-1217. doi:10.1109/TNS.2009.2012859

[8] E. Padovani and S. Pozzi, "MCNP-PoliMi version 1.0," Department of Nuclear Engineering, Polytechnic of Milan, Milan, 2002.

[9] J. R. Lapinskas, “Tension Metastable Fliud Detector for Real-Time Detection of Actinides and Extension to Monitoring of UREX ${ }^{+}$Process Streams," Ph.D. Thesis, Purdue University, West Lafayette, 2010.

[10] X-5 Monte Carlo Team, "MCNP-A General Monte Carlo N-Particle Transport Code, Version 5," Los Alamos National Lab, Los Alamos, 2005. 